\title{
Comparative Evaluation of Push Out Bond Strength of Fiber Post Using One Self-Etch Resin Cement and Two Self-Adhesive Resin Cements - An In-Vitro Study, Punjab Government Dental College and Hospital, Amritsar, Punjab
}

\author{
Baljeet Kumar ${ }^{1}$, Rajinder Kumar² \\ ${ }^{1,2}$ Department of Conservative Dentistry \& Endodontics, Punjab Government \\ Dental College \& Hospital, Amritsar, Punjab, India.
}

\section{ABSTRACT}

\section{BACKGROUND}

Endodontically treated teeth have higher fracture rate due to pronounced coronal destruction. The minimally invasive approach of associating adhesive techniques and posts seems to contribute to a better clinical prognosis for endodontically treated teeth. The purpose of this study was to evaluate the push out bond strength and mode of failure of fiber post using one self-etch resin cement and two selfadhesive resin cements.

\section{METHODS}

Sixty single rooted teeth were selected and decoronated $2 \mathrm{~mm}$ coronal to cementoenamel junction (CEJ). The teeth were prepared and obturated, then divided into three groups of 20 samples each. The post spaces were prepared and relyX fiber posts were cemented in group I with multilink N, in group II with relyX U200 and in group III with Maxcem elite luting cements. The specimens were sectioned into three slices of $2 \pm 0.1 \mathrm{~mm}$ thickness to perform the push out test using universal testing machine. Failure modes were evaluated using scanning electron microscope. Thus, collected data was statistically analysed using one-way analysis of variance test.

\section{RESULTS}

The push out bond strength of group I was significantly higher than that of group II and group III $(\mathrm{P}<0.05)$. No statistically significant difference was found between group II and group III. There was significant difference between coronal, middle and apical thirds in each group. The mode of failures showed $78 \%$ of the samples had adhesive failure while $22 \%$ of the samples had mixed failure.

\section{CONCLUSIONS}

Multilink N was found to be significantly more effective than RelyX U200 and Maxcem elite. Most common type of failure observed was adhesive failure between dentine-cement interface.

\section{KEY WORDS}

Fiber Post, Self-Etch Cement, Self-Adhesive Cement, Bond Strength, Push Out Test
Corresponding Author: Dr. Baljeet Kumar, H.No. 40 J, Rani ka Baag, Amritsar, Punjab, India. E-mail: drbaljeet@gmail.com

DOI: $10.14260 /$ jemds/2021/554

How to Cite This Article:

Kumar B, Kumar R. Comparative evaluation of push out bond strength of fiber post using one self-etch resin cement and two self-adhesive resin cements - an in-vitro study, Punjab Government Dental College and Hospital, Amritsar, Punjab. J Evolution Med Dent Sci 2021;10(33): 2712-2717, DOI:

$10.14260 /$ jemds/2021/554

Submission 04-03-2021,

Peer Review 17-06-2021,

Acceptance 22-06-2021,

Published 16-08-2021.

Copyright (C) 2021 Baljeet Kumar et al. This is an open access article distributed under Creative Commons Attribution License [Attribution 4.0 International (CC BY 4.0)] 


\section{BACKGROUND}

Endodontically treated teeth are more brittle and are more subjected to fracture than vital teeth due to tooth structure loss as a result of caries and access preparation. Due to access preparation, there is more cuspal deflection during function which leads to cusp fracture and microleakage at the restoration margins. ${ }^{1}$ With the removal of pulpal tissue, the neurosensory feedback mechanism is impaired, which may decrease protection of the endodontically treated tooth by masticatory forces. ${ }^{2}$

Coronal tooth preparation leads to loss of tooth structure, which frequently requires post placement inside the root canal. $^{3}$ In this perspective, post plays an imperative role to retain a core which reinforces the coronal restoration. Various materials such as metals, ceramics or fiberreinforced composites have been used to manufacture posts. Due to disadvantages of metallic posts like corrosion, high incidence of root fracture and nickel sensitivity, metal-free post and core system (ceramic and fiber reinforced posts) were introduced. Glass fiber posts were introduced in 1992. Because of similar elastic modulus of fiber post and dentine, which decreases the possibility of root fracture, aesthetically acceptable, does not corrode; facile retrieval in retreatment cases, it can be cemented with a luting agent to avoid friction between the walls of root dentine and the post. 4

Adhesion of the resin cement to the dentine as well as to the post plays an imperative role for retention of fiber posts in root canals. A properly bonded post-dentine unit would have a monoblock like effect which allows for more uniform distribution of stress and prevents both re-infection and vertical cracks in the root. It also reduces dentine-cement interface micro-leakage.5,6 For cementation of fiber post, three systems of adhesive resin cement are used: total-etch, self-etch and self-adhesive systems. The self-etch adhesive system contains acidic primer, which acts both as etch and primer, is the next generation of adhesive system, which is easier to apply as it does not require rinsing and drying procedures in comparison to total-etch system. After self-etch system, self-adhesive system was developed which does not require any treatment of tooth structures. ${ }^{7}$

Since various studies have shown contradictory result as regard to adhesive capability of self-etch adhesive systems and self-adhesive systems, this study was aimed to investigate the influence of one self-etch resin cement and two self-adhesive resin cements on the bond strength of fiber post to root canal dentine, using push-out test setup with universal testing machine and the failure modes of random samples were evaluated with scanning electron microscope at different interfaces.

\section{METHODS}

An in-vitro, experimental study was carried out in the Department of Conservative Dentistry and Endodontics at Punjab Government Dental College \& Hospital, Amritsar, Punjab for a period of 1 year from January 2020 to January 2021.

Objective of this comparative study was to evaluate the push out bond strength and mode of failure of fiber post using one self-etch resin cement and two self-adhesive resin cements. So, for this study sixty, freshly extracted single rooted permanent teeth with single root canal having fully developed apices were selected. Sample size calculation was done by 'Resource Equation' method in which E (sample size) was measured, which is the degree of freedom of analysis o variance (ANOVA). Selected teeth were thoroughly cleaned and stored in $10 \%$ formalin. All the selected teeth were decoronated $2 \mathrm{~mm}$ coronal to cemento-enamel junction with diamond rotary disc leaving approximately $16 \mathrm{~mm}$ of root length. Endodontic access was achieved, with the working length established at $1 \mathrm{~mm}$ short of apex. Root canals were enlarged up to rotary protaper gold F3 file. Intermittent irrigation was done using $1 \mathrm{ml}$ of $3 \%$ sodium hypochlorite. Final irrigation with normal saline was done, followed by drying with paper points and obturated using $\mathrm{AH}$ plus sealer with corresponding F3 gutta percha. Glass ionomer cement was used to temporarily seal the coronal portion of filled roots. The prepared samples were randomly divided into three groups of 20 samples each, based on luting cement used;

- Group I - For application of Multilink N (Ivoclar Vivadent AG, Schaan, Liechtenstein)

- Group II - For application of RelyX U200 (3M ESPE, Neuss, Germany)

- Group III - For application of Maxcem Elite (Kerr Corporation, Orange CA, USA)

\section{Post Space Preparation}

The sealing material was removed. The post spaces were prepared with a pre-calibrated size-1 drill which corresponds to the size of RelyX Fiber Post (3M ESPE, France) \# 1 (1.3 mm in diameter). $17 \%$ ethylene diamine tetra acetic acid (EDTA) solution was used to rinse the canal for 1 minute. The depths of the post-spaces preparation were $11 \mathrm{~mm}$ from the coronal reference point resulting in $4 \mathrm{~mm}$ of apical sealing. After preparation, the root canals were cleaned with distilled water and gently dried with absorbent paper points. The posts were cleaned with $70 \%$ ethanol and dried with absorbent paper towels.

\section{Fiber Post Luting Procedure}

Fiber posts were luted with Multilink N in group I, with RelyX U200 in group II and with Maxcem Elite in group III as per manufacturer's instruction respectively.

To prevent dehydration of the specimens, they were stored in a flower sponge, slightly damped with deionized water for 1 week at room temperature, until the push-out test was performed.

\section{Push Out Bond Strength Evaluation}

For evaluation of push out bond strength, a thin slice pushout test was used. All the specimens were mounted in acrylic resin. Each specimen was sectioned horizontally by using diamond rotary disc mounted on mandrel in straight handpiece under water cooling at slow speed to produce three $2 \pm 0.1 \mathrm{~mm}$ thick post-dentin sections from CEJ to apical third (cervical, middle, and apical). A digital caliper was used to determine exact thickness of each slice. Universal testing machine was used to apply load in an apical to cervical 
direction at $1 \mathrm{~mm} / \mathrm{min}$ crosshead speed until the post gets dislodged. The bond strength ( $\mathrm{MPa}$ ) was calculated as:

$\mathrm{MPa}^{*}=\mathrm{N} / 2 \mathrm{Xprh}$

Where $\mathrm{p}=3.14, \mathrm{r}$ was the radius of the post segment $(\mathrm{mm})$, and $\mathrm{h}$ was the thickness of the post segment $(\mathrm{mm})$. ${ }^{*} \mathrm{MPa}$ - Megapascal.

Scanning Electron Microscope (SEM) Analysis Five random samples from each group with their corresponding posts were prepared for scanning under electron microscope (SUPRA 55VP, ZEISS, Germany) analysis at X100 magnification. Demineralization of selected samples with phosphoric acid (37\%) for 20 seconds was done. After that, samples were mounted on stubs and gold sputter- coating was done. The mode of failures were inspected for different interfaces and classified as follows: A - adhesive failures between the dentine and the resin cement; B adhesive failures between the post and the resin cement; $\mathrm{C}$ cohesive failures within the post; $\mathrm{M}$ - mixed failures (between the dentine-resin cement and between the post cement).

\section{Statistical Analysis}

The collected data of push-out bond strength was subjected to statistical analysis using one-way ANOVA test of variance (Statistical Package for Social Science software, Chicago, IL, USA) with Tukey post-hoc test for multiple comparisons.
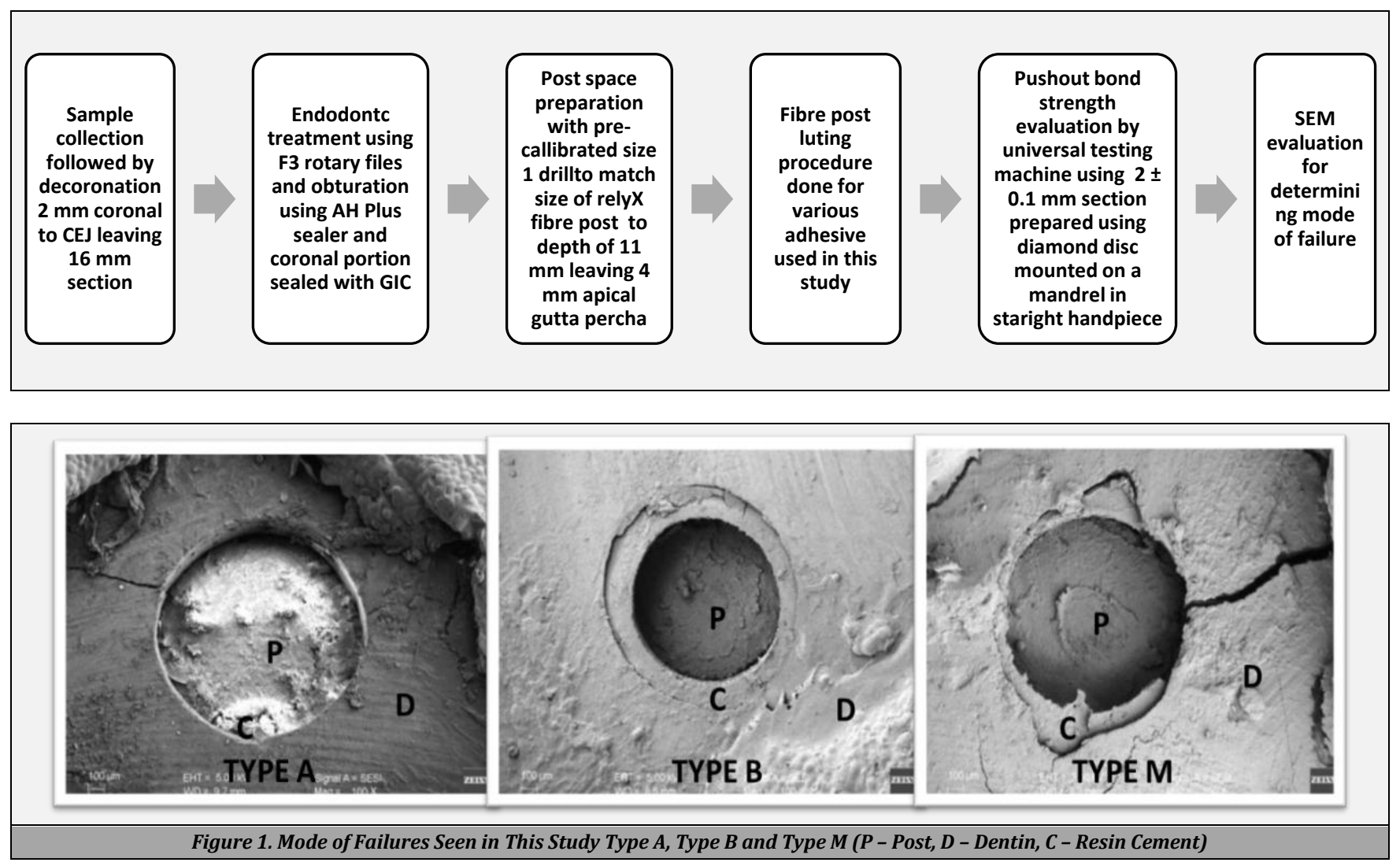

\begin{tabular}{|cccc|}
\hline Experimental Groups & Cervical & Middle & Apical \\
Group I & 12.06 & 10.65 & 9.05 \\
Group II & 10.08 & 8.87 & 7.55 \\
Group III & 9.58 & 8.41 & 7.14 \\
\hline Table 1. Mean Values of Push-Out Bond Strength in MPA \\
\hline
\end{tabular}

\begin{tabular}{|c|c|c|c|c|c|c|}
\hline \multirow[t]{2}{*}{ Level } & \multirow{2}{*}{ Groups } & \multirow{2}{*}{$\begin{array}{c}\text { Mean } \\
\text { Difference }\end{array}$} & \multirow{2}{*}{$\begin{array}{l}\text { Standard } \\
\text { Error }\end{array}$} & \multicolumn{2}{|c|}{$\begin{array}{l}95 \% \text { Confidence } \\
\text { Interval }\end{array}$} & \multirow[t]{2}{*}{ P Value } \\
\hline & & & & Lower & Upper & \\
\hline \multirow{3}{*}{ Coronal } & I / II & 1.979 & 0.236 & 1.410 & 2.548 & 0.001 \\
\hline & II / III & 0.525 & 0.236 & -0.043 & 1.094 & 0.076 \\
\hline & I / III & 2.505 & 0.236 & 1.935 & 3.074 & 0.001 \\
\hline \multirow{3}{*}{ Middle } & I / II & 1.782 & 0.261 & 1.152 & 2.363 & 0.001 \\
\hline & II / III & 0.523 & 0.261 & -0.105 & 1.152 & 0.121 \\
\hline & I / III & 2.305 & 0.261 & 1.673 & 2.934 & 0.001 \\
\hline \multirow{3}{*}{ Apical } & I / II & 1.502 & 0.247 & 0.907 & 2.096 & 0.001 \\
\hline & II / III & 0.450 & 0.247 & -0.144 & 1.045 & 0.171 \\
\hline & I / III & 1.952 & 0.247 & 1.358 & 2.547 & 0.001 \\
\hline & Ta & $\begin{array}{l}\text { 2. Inter } \\
\text { d Stren }\end{array}$ & $\begin{array}{l}\text { oup Mu } \\
\text { at Corc }\end{array}$ & le Com & $\begin{array}{l}\text { son of } \\
\text { d Apica }\end{array}$ & \\
\hline
\end{tabular}

\begin{tabular}{|ccccc|}
\hline Groups & Type A & Type B & Type C & Type M \\
Group I & $60 \%$ & $27 \%$ & $0 \%$ & $13 \%$ \\
Group II & $60 \%$ & $13 \%$ & $0 \%$ & $27 \%$ \\
Group III & $53 \%$ & $20 \%$ & $0 \%$ & $27 \%$ \\
\hline Table 3. Mode of Failure at Different Interfaces & Observed & Under SEM \\
\hline
\end{tabular}

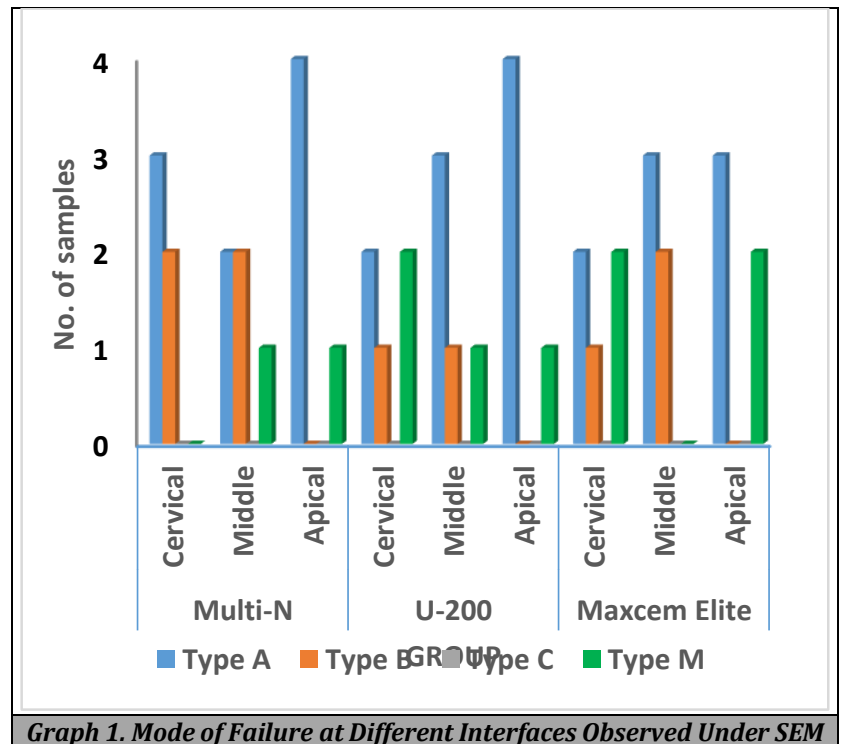

Graph 1. Mode of Failure at Different Interfaces Observed Under SEM 
- $\quad$ Group I $60 \% \quad 27 \% \quad 0 \% \quad 13 \%$

- Group II $60 \% \quad 13 \% \quad 0 \% \quad 27 \%$

- $\quad$ Group III $53 \% \quad 20 \% \quad 0 \% \quad 27 \%$

\section{Ethical Statement}

The research was conducted in Department of Conservative Dentistry and Endodontics and was approved by the ethics committee of Punjab Government Dental College and Hospital, Amritsar (Baba Farid University of Health Sciences, Faridkot)

\section{RESULTS}

The statistical analysis by using ANOVA (Statistical Package for Social Science software, Chicago, IL, USA) with Tukey's post-hoc HSD test and $P$ value showed statistically significant difference in push-out bond strength between group I and group II, between group I and group III $(\mathrm{P}<0.05)$. Push-out bond strength between group II and group III was not significantly different $(\mathrm{P}>0.05)$. Statistically significant difference was found between coronal and middle, middle and apical, coronal and apical thirds in each group $(\mathrm{P}<0.05)$.

SEM analysis revealed that $78 \%$ of the samples of all experimental groups showed adhesive failure (Type A and Type B) while $22 \%$ of the samples showed mixed failure (Type M). Among the samples which showed adhesive failure, $58 \%$ of samples showed failure between dentine and luting material, while $20 \%$ adhesive failure were observed between the post and the luting material. None of the samples showed cohesive failure.

\section{DISCUSSION}

Endodontically treated teeth with significant coronal structure loss were restored with cast metal post and core from decades. Metal posts have been associated with irreparable root fractures. This is primarily due to the elastic modulus of metal being higher than that of root dentin. ${ }^{8}$ Since the 1990s, post with an elastic modulus closer to dentine such as prefabricated carbon fiber posts were introduced. After that, fiber-reinforced composite posts were invented and have been increasingly used for restoration. ${ }^{9}$

RelyX fiber posts can be primarily ascribed because of an elastic modulus that is similar to that of dentin and of good esthetics. ${ }^{3}$ RelyX fiber posts are tapered in shape and its configuration resembles the anatomical structure of the root. These posts fit in post spaces quite well, because of similar drills of same size and shape provided by the manufacturer. ${ }^{4}$

For cementation of fiber post, one self-etch and two selfadhesive cements were used in this study. The self-etch cement is a self-cure resin with a light curing option in which the main reaction is chemical and its polymerization can be further enhanced achieved by light cure. ${ }^{10}$ Whereas, the basic adhesion mechanism of self-adhesive resin cements depends upon micromechanical retention and chemical interactivity between hydroxyapatite and the monomer acidic groups. Dual adhesive monomers such as 10 - methacryloyloxydecyl dihydrogen phosphate (10 - MDP) demonstrate durable chemical bond integrity because it binds chemically to the calcium atoms in the dentin. ${ }^{11}$

Literature shows that there is wide variations in thickness $(1-4 \mathrm{~mm})$ of samples, however in present study 2 $\pm 0.1 \mathrm{~mm}$ thickness is standardized because it presents less chance of over estimation of results and lower friction areas. $^{12}$ The push out test is better for bond strength evaluation because it is true shear test as the fracture occurs parallel to the dentine bonding interface. ${ }^{13}$

Results of this study demonstrated that bonding strength values of the self-curing resin material is higher than the dual cure resin materials. This might be attributed to several factors, one of them is that the dual cure resin cement is a light polymerized adhesive, its bonding strength value is compromised at the apical and middle regions of the root where the curing light might not reach, while the self-cure cement (Multilink $\mathrm{N}$ ) is a self-cure resin with a light curing option in which the main reaction is chemical and its polymerization can be further enhanced and achieved by the light cure. ${ }^{10}$ Moreover, Multilink $\mathrm{N}$ adhesive system contain Primer A and Primer B, functioning both as etch and primer, which do not require rinsing and drying procedure. The findings of this study coincide with study by Behr et al.14 which revealed that the self-activating system presents uniform resin tags and resin-dentine inter-diffusion zone formation with root canal as compare to dual-cure system, while conflict the study by Da Silva et al. ${ }^{15}$ who revealed in their study that the bonding strength values of dual cure resin is higher than of self-cure resin cement. This might be due to methodological variation. Additionally, the Multilink $\mathrm{N}$ requires silanization of the post with Monobond S which serves as a bonding agent and creates a durable chemical bond between the fiber post and the resin cement. This is in agreement with Goracci et al. ${ }^{16}$ who stated that bond strength between the resin cement and fiber post is significantly improved when using silane, while in disagreement with Bitter et al. ${ }^{17}$ who stated that silanization do not significantly affect push-out bond strength of the resin cement to fiber posts.

Whereas self-adhesive RelyX U200 resin cement used in this study does not require surface treatment of dentine, and its mechanism of action is based on chemical interaction with hydroxyapatite. ${ }^{18}$ RelyX U200 includes acidic and hydrophilic monomers, which demineralize and infiltrate the tooth surface simultaneously promoting chemical interaction and micromechanical retention which provide adequate bond strength to dentine. ${ }^{19}$ This study evaluated that Maxcem Elite has self-adhesive property because of glyceroldimethacrylate dihydrogen phosphate (GPDM) and various adhesive monomers which improve wettability. As Maxcem Elite also contains 2 - hydroxyethyl methacrylate (HEMA) which renders the resin prone to water sorption. Moreover, porosity of this cement might provide a pathway for acidic products or enzymes, which leads to activation of degradation process. ${ }^{20}$

Different regions of the root canal have significant influence on bond strength, that is, cervical third has the highest value because of a greater number of dentinal tubules as compared to apical third which has less dentinal tubules. The accessibility of cervical portion is another factor for higher bond strength as it enables etching and application of 
adhesive agent thoroughly. Whereas, in apical third, reduction of strength is due to decrease in the effectiveness of light cure at longer distances and increased difficulty of distribution of resin cement because of gutta-percha and sealer traces or void formation after preparation of post space. The other factor for lower bond strength may be due to high configuration factor stresses (which may exceed 200) generated within narrow, long post spaces leading to numerous interfacial gaps. ${ }^{21}$

After SEM analysis, it has been inferred that type A mode of failure was predominant due to polymerization stresses occurring at the interface of dentine and the resin cements. Type B mode of failure may occur due to absence of a chemical union between and methacrylate-based resins and epoxy resin-based post. Thus, proposed use of silane is recommended to improve bonding, ${ }^{22}$ although Perdigao et al. ${ }^{23}$ reported that silane coupling agent do not increase bond strength. There was no sample which showed cohesive failure (Type C). This is attributed to the properties of the individual material used for this study.

\section{CONCLUSIONS}

Within the limitations of this study, it can be concluded that the values of mean push out bond strength of group I in which Multilink N was used to lute fiber post, showed higher bond strength than group II in which RelyX U200 and group III in which Maxcem Elite was used. Most common failure was observed in this study was adhesive failure at the dentinecement interface.

\section{Limitations of This Study}

Before any definitive conclusion can be drawn, a clinical evaluation with large number of samples and more extensive research with a definite data distribution should be done to evaluate both adhesive system in future.

Data sharing statement provided by the authors is available with the full text of this article at jemds.com.

Financial or other competing interests: None.

Disclosure forms provided by the authors are available with the full text of this article at jemds.com.

\section{REFERENCES}

[1] Pantvisai P, Messer HH. Cuspal deflection in molars in relation to endodontic and restorative procedures. Journal of Endodontics 1995;21(2):57-61.

[2] Robbins JW. Restoration of the endodontically treated tooth. Dental Clinics of North America 2002;46(2):36784.

[3] Desai P, Maiti A, Dutta K, et al. Comparison of push out bond strength of customizable fiber post using two self adhesive resin cement - an in - vitro study. Adv Dent and Oral Health 2016;2(1):1-6.

[4] Khan S, Pirvani M, Malik S. Evaluation of push out bond strength of a dual - cure self - adhesive resin - cement with fiber post systems and dentine. J Pak Dent Assoc 2015;24(1):28-34.

[5] Bhalla S, Shafia, Bali D, et al. To evaluate the influence of pre - treatment of fiber post on bond strength between fiber post and root dentin in push out test - an invitro study. Annals of International Medical and Dental Research 2019;5(3):38-42.

[6] Uzun İH, Malkoç MA, Keleş A, et al. 3D micro - CT analysis of void formations and push - out bonding strength of resin cements used for fiber post cementation. The Journal of Advanced Prosthodontics 2016;8(2):101-9.

[7] Theodor Y, Koesmaningati H, Gita F. Adhesive capability of total-etch, self-etch and self-adhesive systems for fiber post cementation. Journal of Physics: Conference Series 2017;884(1):012098.

[8] Bazzo JF, Pedriali MBBP, Guiraldo RD, et al. Push - out bond strength of different translucent fiber posts cemented with self - adhesive resin cement. Journal of Conservative Dentistry 2016;19(6):583-6.

[9] Baldea B, Furtos G, Antal M, et al. Push-out bond strength and SEM analysis of two self-adhesive resin cements: an in vitro study. Journal of Dental Sciences 2013;8(3):296305.

[10] Al-Azzawi HJ. Comparison of regional bond strength of post space of fiber-reinforced post luted with two types of cements at different testing times. Journal of Baghdad College of Dentistry 2013;25(3):19-23.

[11] Özlek E, Neelakantan P, Matinlinna JP, et al. Adhesion of two new glass fiber post systems cemented with selfadhesive resin cements. Dentistry Journal 2019;7(3):80.

[12] Rajan I, Ravi V, Sivakumar AA, et al. Assessment of the bond strength and mode of failure of glass fibre posts luted using two different adhesives: an in vitro analysis. Journal of Operative Dentistry \& Endodontics 2018;3(2):57-62.

[13] Das NS, Rupesh PL, Kataraki B, et al. Evaluation and comparison of the tensile bond strength of two different luting agents on an endodontic fiber post after two different surface treatments- an in vitro study. Int J Adv Res 2019;7(3):1201-26.

[14] Behr M, Rosentritt M, Regnet T, et al. Marginal adaptation in dentin of a self-adhesive universal resin cement compared with well-tried systems. Dental Materials 2004;20(2):191-7.

[15] Silva RA, Coutinho M, Cardozo PI, et al. Conventional dual-cure versus self-adhesive resin cements in dentin bond integrity. Journal of Applied Oral Science 2011;19(4):355-62.

[16] Goracci C, Raffaelli O, Monticelli F, et al. The adhesion between prefabricated FRC posts and composite resin cores: microtensile bond strength with and without postsilanization. Dental Materials 2005;21(5):437-44.

[17] Bitter K, Meyer-Lückel H, Priehn K, et al. Bond strengths of resin cements to fiber-reinforced composite posts. American Journal of Dentistry 2006;19(3):138-42.

[18] Pelegrine RA, Paulillo LAMS, Kato AS, et al. Effect of endodontic retreatment on push-out bond strength and quality of fiber postbonding interface of resin cements. The Journal of Contemporary Dental Practice 2016;17(1):42-8.

[19] Mendes M, França FM, Basting RT, et al. Long-term bond strength of fiber posts cement to dentin with self- 
adhesive or conventional resin cements. Journal of Adhesion Science and Technology 2017;31(9):977-87.

[20] Cantoro A, Goracci C, Vichi A, et al. Retentive strength and sealing ability of new self-adhesive resin cements in fiber post luting. Dental Materials 2011;27(10):e197204.

[21] Erdemir U, Mumcu E, Topcu FT, et al. Micro push-out bond strengths of 2 fiber post types luted using different adhesive strategies. Oral Surgery, Oral Medicine, Oral
Pathology, Oral Radiology and Endodontology 2010;110(4):534-44.

[22] Soares CJ, Pereira JC, Valdivia ADCM, et al. Influence of resin cement and post configuration on bond strength to root dentine. International Endodontic Journal 2012;45(2):136-45.

[23] Perdigão J, Gomes G, Lee IK. The effect of silane on the bond strengths of fiber posts. Dental Materials 2006;22(8):752-8. 